\title{
Low 30-day mortality in South African orthopaedic patients undergoing surgery at an academic hospital during the first wave of the COVID-19 pandemic: It was safe to perform orthopaedic procedures at our hospital during the first COVID-19 peak
}

\author{
R Waters, ${ }^{1,2,3} \mathrm{MSc} ; \mathbf{R}$ Dey, ${ }^{1,2,4} \mathrm{PhD} ;$ M Laubscher, ${ }^{1,2} \mathrm{MB}$ ChB, Dip PEC (SA), FC Orth (SA), MMed (Orth); \\ R Dunn, ${ }^{1,2}$ MB ChB, FCS (SA) Orth, MMed (Orth); S Maqungo, ${ }^{1,2}$ MB ChB, FC Orth (SA), MMed (Orth), PG Dip HPE; \\ G McCollum, ${ }^{1,2}$ MB ChB, MMed (Orth), FC Orth (SA); M Nortje, ${ }^{1,2}$ MB ChB, FC Orth (SA), MMed (Orth); \\ S Roche, ${ }^{1,2}$ MB ChB, FC Orth (SA), LMCC (Canada) (Orth); T Hilton, ${ }^{2}$ MB ChB, DA (SA), Dip PEC (SA), FC Orth (SA), MMed (Orth); \\ W Mugla, ${ }^{1,2}$ MB ChB, FC Orth (SA); M Held ${ }^{1,2}$ MD, MMed (Orth) , FC Orth (SA), PhD, MBA

\begin{abstract}
${ }^{1}$ Orthopaedic Research Unit, Division of Orthopaedic Surgery, Faculty of Health Sciences, University of Cape Town, South Africa
${ }^{2}$ Division of Orthopaedic Surgery, Faculty of Health Sciences, University of Cape Town, South Africa

${ }^{3}$ Wellcome Centre for Infectious Disease Research in Africa (CIDRI-Africa), Institute of Infectious Disease and Molecular Medicine, Faculty of Health Sciences, University of Cape Town, South Africa

${ }^{4}$ Division of Biomedical Engineering, Department of Human Biology, Faculty of Health Sciences, University of Cape Town, South Africa
\end{abstract}

Corresponding author: $R$ Waters (WTRROB005@myuct.ac.za)

\begin{abstract}
Background. Initial local and global evidence suggests that SARS-CoV-2-infected patients who undergo surgery, and those who become infected perioperatively, have an increased mortality risk post surgery.

Objectives. To analyse and describe the 30-day mortality, presurgical COVID-19 status and hospital-acquired SARS-CoV-2 infection rates of patients, both SARS-CoV-2-positive and negative, undergoing orthopaedic surgery at a tertiary academic hospital in South Africa (SA) during the first COVID-19 peak.

Methods. This single-centre, observational, prospective study included patients who underwent orthopaedic procedures from 1 April 2020 (beginning of the COVID-19 case increase in SA) to 31 July 2020 (first COVID-19 peak in SA). All patients were screened for COVID19 and were confirmed positive if they had a positive laboratory quantitative polymerase chain reaction test for SARS-CoV-2 RNA on a nasopharyngeal or oral swab. Thirty-day mortality, presurgical COVID-19 status and hospital-acquired SARS-CoV-2 infection were assessed. Results. Overall, a total of 433 operations were performed on 346 patients during the timeframe. Of these patients, $65.9 \%(n=228)$ were male and $34.1 \%(n=118)$ were female. The mean (standard deviation) age was 42.5 (16.8) years (range 9 - 89). Of the patients, $5(1.4 \%)$ were identified as COVID-19 patients under investigation (PUI) on admission and tested positive for SARS-CoV-2 before surgery, and $1(0.3 \%)$ contracted SARS-CoV-2 perioperatively; all survived 30 days post surgery. Twenty-nine patients were lost to follow-up, and data were missing for 6 patients. The final analysis was performed excluding these 35 patients. Of the 311 patients included in the final 30-day mortality analysis, 303 (97\%) had a follow-up observation $\geq 30$ days after the operation. The overall 30 -day mortality for these patients was $2.5 \%$ ( $n=8$ deaths). None of the recorded deaths were of screened COVID-19 PUI.

Conclusions. We report a low 30-day mortality rate of $2.5 \%(n=8)$ for patients undergoing orthopaedic surgery at our hospital during the first COVID-19 peak. None of the deaths were COVID-19 related, and all patients who tested SARS-CoV-2-positive, before or after surgery, survived. Our overall 30-day mortality rate correlates with several other reports of orthopaedic centres analysing over similar timeframes during the first peak of the COVID-19 pandemic. Regarding mortality and SARS-CoV-2 infection risk, we can conclude that with the appropriate measures taken, it was safe to undergo orthopaedic procedures at our hospital during the first peak of the COVID-19 pandemic in SA.
\end{abstract}

S Afr Med J 2021;111(8):747-752. https://doi.org/10.7196/SAMJ.2021.v111i8.15766

South Africa (SA) remains in a national state of disaster due to COVID-19 and the ongoing detection of active SARS-CoV-2 cases in our population, mainly driven by the local Beta variant 501Y.V2. Our nation's recorded recovery rate remains high at $92 \% \cdot{ }^{[1]}$ Groote Schuur Hospital (GSH), one of the two state academic hospitals in our area, services patients from vulnerable communities, where infectious diseases such as tuberculosis and HIV continue to dominate the health landscape and multiple comorbidities exist, posing a potential risk for the development of more severe forms of COVID-19- associated illness. Many of our orthopaedic patients are from a low socioeconomic background and low-income households, associated with potential vulnerabilities.

At GSH, both elective and non-urgent surgeries were cancelled during the initial phases of the hard lockdown, 26 March to the end of May 2020. ${ }^{[2]}$ After the initial hard lockdown, we resumed semi-urgent surgeries in lower-risk patients. We used the medically necessary, time-sensitive procedures (MeNTS) scoring system to help select the most appropriate orthopaedic patients to perform surgery 
on. ${ }^{[3]}$ At the time of writing, elective and non-urgent surgeries were actively resuming and patients were once again returning to hospitals to seek diagnosis and treatment. It remains a top priority to continue ensuring that both patients and healthcare workers are protected from contracting SARS$\mathrm{CoV}-2$ in the course of patient treatment. A primary focus should be how to safely and fully reinstate elective and non-urgent operating amid the current pandemic and the devastating 'waves'. Here, it is of key importance to provide evidence regarding the safety of surgery in hospitals with high SARS-CoV-2 risk. It is also valuable to assess how COVID-19 may affect surgical outcomes of patients treated during this time, as initial global evidence has suggested that COVID-19-positive patients who undergo surgery have significantly increased mortality and risk of major complications post surgery. ${ }^{[4-9]}$ There is limited evidence of the impact of COVID-19 on the mortality of orthopaedic patients undergoing surgery in resource-limited settings.

\section{Objectives}

To assess the pre- and perioperative COVID19 status and 30-day mortality of all patients who underwent orthopaedic surgery during the first peak of the COVID-19 pandemic in our tertiary academic hospital. To our knowledge, no reports on the 30-day mortality of orthopaedic patients in a level 1 orthopaedic trauma unit in an SA environment are available.

\section{Methods}

\section{Study design and data source}

After obtaining ethical (ref. no. HREC 250/ 2020) and hospital institutional approval, orthopaedic surgical case data for the timeline 1 April 2020 - 31 July 2020 were extracted from a prospectively collected orthopaedic REDCap database, while maintaining patient confidentiality. All patients presenting to the orthopaedic department were screened for COVID-19 using standardised screening questions and identified as patients under investigation (PUI) if they exhibited COVID-19-related signs and symptoms including a history of fever, shortness of breath, cough, sore throat and/or loss of smell and taste, and/or if they had recently travelled abroad or had contact with an individual with suspected or confirmed SARS-CoV-2. Only the COVID19 PUI underwent a nasopharyngeal or oral swab for detection of SARS-CoV-2 RNA by quantitative polymerase chain reaction (PCR), as per hospital guidelines.
We attempted to contact by telephone all identified orthopaedic patients $(n=346)$ who underwent surgery during the study period. Patients who were successfully contacted provided telephonic consent for the investigators to assess their COVID-19 status and the status of their household members, and if applicable, information on patient mortality. In addition, all identified patients were screened using the Picture Archiving and Communication System (PACS) imaging database to assess the date of latest imaging results for the purpose of understanding patient 30-day mortality. Concurrently, all 346 patients were also screened using the National Health Laboratory Service (NHLS)-LABTRAK (TrakCare) system, both for latest result dates to assess 30-day mortality, for those unsuccessful on the PACS system, and for SARS-CoV-2 PCR results. Patient folders were extracted and Clinicom was accessed for patients who had neither a PACS nor an NHLS follow-up date post surgery, to assess last-seen dates and 30-day mortality, if known to be deceased.

\section{Statistical analysis}

Patient demographic data were summarised using descriptive statistics, including means and ranges, where applicable.

\section{Results}

A total of 433 orthopaedic surgical procedures were performed on 346 patients from 1 April to 31 July 2020 . All 346 patients were screened for COVID-19 signs and symptoms on admission. Of these, 36 (10.4\%) were identified as PUI and underwent a nasopharyngeal swab for diagnostic testing by the NHLS using a SARS-CoV-2 PCR; 5 (13.9\%) were confirmed SARS-CoV-2-positive, 28 (77.8\%) were confirmed SARS-CoV-2-negative, and $3(8.3 \%)$ had inconclusive results or tests rejected due to incorrect sample labelling (Fig. 1 and Table 1). Two hundred and eightyone orthopaedic patients were not swabbed for SARS-CoV-2 because they screened negative for COVID-19 high risk according to the hospital swabbing guidelines at the time. Assessment of presurgical COVID-19 status for 29 patients was unsuccessful owing to incorrect recording of folder numbers on the REDCap database or lack of 2020 data on the TrakCare system (Fig. 1).

Of the patient cohort, $65.9 \%(n=228)$ were male. The mean (standard deviation) age of the entire patient cohort was 42.5 (16.8) years (range 9 - 89) (Table 1). The ages of 2 patients were unrecorded or unavailable owing to incorrect folder numbers recorded on the REDCap database.

Of the patients, $56.9 \%$ underwent surgery for orthopaedic trauma. There was an equal spread among the rest of the orthopaedic surgical firms, with the exception of spine trauma (2.3\%), foot and ankle (1.4\%) and paediatric non-trauma (0.3\%) (Table 1 ).

Available data for our orthopaedic surgical cohort for the 30-day mortality assessment are shown in Fig. 2. A total of 35 patients were excluded from the final analysis (dotted lineframed boxes) because the patient hospital folder number was incorrectly recorded on the orthopaedic REDCap database $(n=6)$ or the patients were lost to follow-up (LTFU) post surgery $(n=29)$. Of the remaining 311 patients, 303 (97.4\%) had a $\geq 30$-day followup date (PACS, NHLS, telephonic followup, folder retrieval), of whom 177 (58.4\%) were successfully contacted telephonically. The overall 30-day mortality for our patient

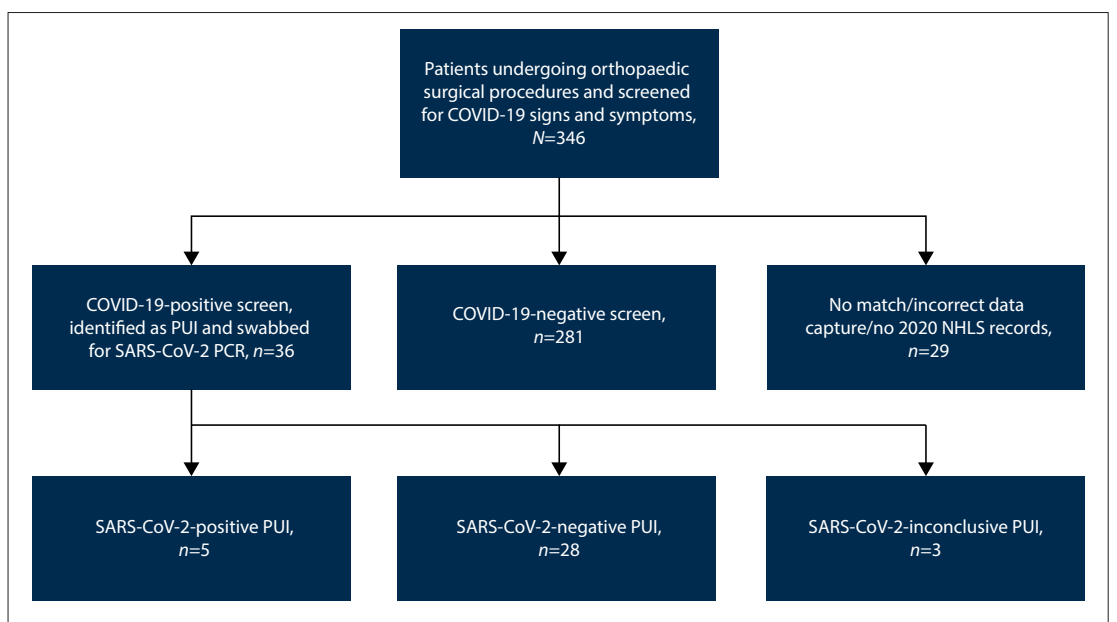

Fig. 1. Flow chart showing screening of our orthopaedic surgical cohort for COVID-19 signs and symptoms on admission. ( $P U I=$ patients under investigation $; P C R=$ polymerase chain reaction; NHLS = National Health Laboratory Service. $)$ 


\begin{tabular}{|c|c|c|c|}
\hline Variable & Overall & Non-trauma ${ }^{*}$ & Trauma \\
\hline \multicolumn{4}{|l|}{ Gender, $n(\%)$} \\
\hline Total & $346(100)$ & $149(43.1)$ & $197(56.9)$ \\
\hline Male & $228(65.9)$ & $89(39.0)$ & $139(61.0)$ \\
\hline Female & $118(34.1)$ & $60(50.8)$ & $58(49.2)$ \\
\hline Age (years), mean (SD, range) & $42.5(16.8,9-89)$ & $44.02(17.61,9-89)$ & $41.26(16.1,14-88)^{\dagger}$ \\
\hline \multicolumn{4}{|l|}{ Surgical procedures per surgical firm, \% } \\
\hline Arthroplasty & 9.0 & - & - \\
\hline Foot/ankle & 1.4 & - & - \\
\hline Knee & 6.6 & - & - \\
\hline Limb reconstruction unit & 7.5 & - & - \\
\hline Oncology & 5.2 & - & - \\
\hline Paediatric non-trauma & 0.3 & - & - \\
\hline Spine & 6.6 & - & - \\
\hline Spine trauma & 2.3 & & . \\
\hline Trauma & 56.9 & - & - \\
\hline Upper limb & 4.0 & - & - \\
\hline \multicolumn{4}{|l|}{ 30-day mortality characteristics } \\
\hline Total, $n$ (\% of total patient cohort) & $8(2.5)$ & $1(12.5)$ & $7(87.5)$ \\
\hline COVID-19 PUI on admission, $n$ & 0 & 0 & 0 \\
\hline Age (years), mean (SD, range) & $57.5(18.02,30-83)$ & - & $59.14(18.8,30-83)$ \\
\hline Procedures, $n$ (\% of total patient cohort) & $8(2.5)$ & $1(12.5)$ & $7(87.5)$ \\
\hline ORIF (nail), $n$ & 2 & - & 2 \\
\hline ORIF (plate), $n$ & 1 & - & 1 \\
\hline MUA, $n$ & 1 & - & 1 \\
\hline Cervical spine fusion, $n$ & 1 & 1 & - \\
\hline Soft-tissue debridement, $n$ & 1 & - & 1 \\
\hline Removal of hardware, $n$ & 1 & - & 1 \\
\hline Soft-tissue shoulder arthrotomy, $n$ & 1 & - & 1 \\
\hline \multicolumn{4}{|c|}{ Pre- and perioperative SARS-CoV-2-positive patient characteristics and surgery detail } \\
\hline $\begin{array}{l}\text { Preoperative SARS-COV- } 2 \text { confirmed infection, } \\
n \text { (\% of total patient cohort) }\end{array}$ & $5(1.4)$ & - & $5(100)$ \\
\hline Age (years), mean (SD, range) & $42.2(15.97,21-65)$ & - & $42.2(15.97,21-65)$ \\
\hline Procedures, $n$ (\% of total patient cohort) & $5(1.4)$ & - & $5(100)$ \\
\hline ORIF (nail), $n$ & 2 & - & 2 \\
\hline ORIF (plate), $n$ & 1 & - & 1 \\
\hline Amputation, $n$ & 1 & - & 1 \\
\hline Cemented bipolar, $n$ & 1 & - & 1 \\
\hline $\begin{array}{l}\text { Perioperative SARS-COV- } 2 \text { confirmed infection, } \\
n \text { ( } \% \text { of total patient cohort) }\end{array}$ & $1(0.3)$ & - & $1(100)$ \\
\hline Procedure - ORIF (plate), $n$ ( $\%$ of total patient cohort) & $1(0.3)$ & - & $1(100)$ \\
\hline
\end{tabular}

cohort was $2.5 \%$ ( $n=8$ deaths). Of the 8 patients who died during the study period, $87.5 \%(n=7)$ underwent trauma-related surgery and 1 patient underwent a cervical spine fusion. There was an equal ratio between males and females who died postoperatively. None of the recorded deaths were of COVID-19 PUI on admission. Of the patients confirmed positive for SARS-CoV-2 who underwent surgery during the timeframe, $80.0 \%$ were male. The most common procedure performed was open reduction and internal fixation of a fracture. One orthopaedic trauma patient $(0.3 \%)$ contracted SARSCoV-2 perioperatively (Table 1 ). After 30 days, $100 \%$ survival was recorded for all patients who were admitted with SARS-CoV-2 or contracted it during the postoperative period.

\section{Discussion}

To our knowledge, this is the first SA study to have analysed and described the 30-day mortality and pre- and perioperative SARS$\mathrm{CoV}-2$ infection status of orthopaedic patients undergoing surgery at a tertiary academic hospital during the first wave of the COVID-19 pandemic.

As we actively resume elective and non-urgent surgical procedures between waves of the COVID-19 pandemic, it is important to acknowledge the continued SARS-CoV-2-exposure risks, both to patients and to surgical staff. Most of the patients in our study cohort underwent surgery for trauma-related injuries, which inherently carries an increased risk to the patient. The risks due to SARS- 


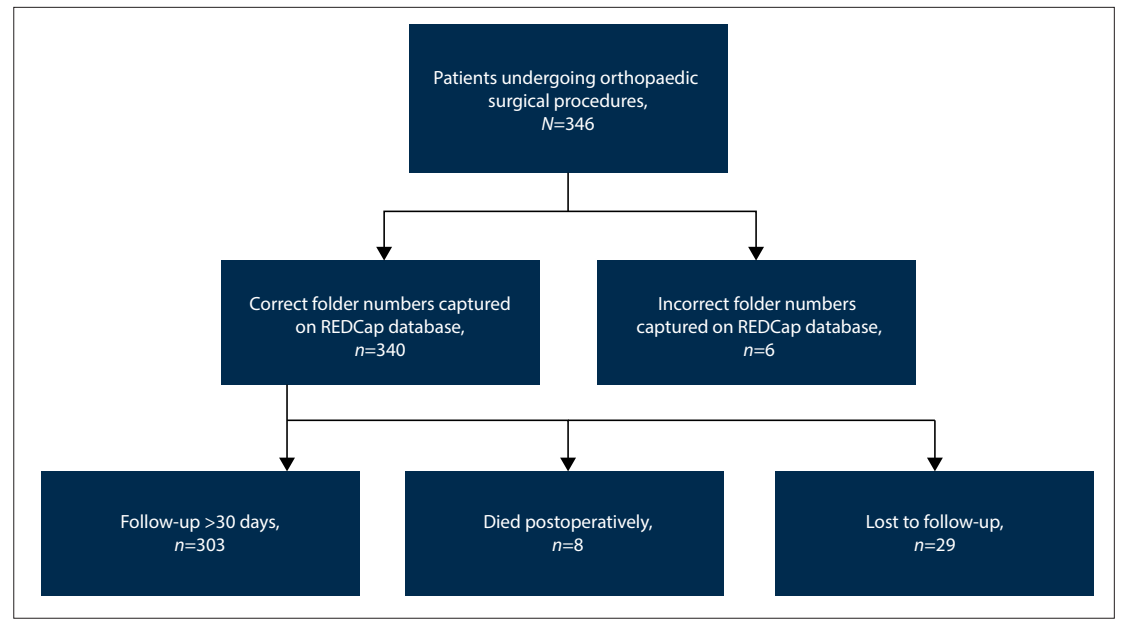

Fig. 2. Flow chart showing available data for our orthopaedic surgical cohort for the 30-day mortality assessment.

CoV-2 infection, in SARS-CoV-2 confirmed positive patients on admission, may compound this general risk, and outcomes could be unfavourable for the patient. Despite these risks, all our SARS-CoV-2 confirmed positive patients undergoing surgery survived 30 days post surgery. Of our cases, $43.1 \%$ were non-trauma, as we were actively conducting urgent and semiurgent orthopaedic procedures during this time.

Globally, several orthopaedic units have assessed the 30-day mortality and SARSCoV-2 infection rates of patients undergoing procedures during their respective first peaks of the COVID-19 pandemic. Most of the study sites, including in the UK, Europe and the USA, experienced their first COVID-19 peak in March and April 2020, earlier than that experienced in SA in July 2020. We have summarised and described the findings in Table 2. The low 30-day mortality rate of our patient cohort during the first COVID-19 peak is similar to figures published by Sobti et al., ${ }^{[10]}$ Karayiannis et al., ${ }^{[11]}$ Price et al. ${ }^{[12]}$ Hope et al. ${ }^{[13]}$ and Giorgi et al. ${ }^{[14]}$ (2.3\% v. $4.3 \%, 1.9 \%, 3 \%, 4 \%$ and $4.6 \%$, respectively), and is considerably lower than mortality rates reported in other regions in the UK and other developing countries $(11.76 \%-53 \%))^{[5,7-9,13]}$ The specific reasons for this lower mortality rate are not clear from our study, but may be attributed to an overall younger population undergoing surgery in our academic hospital and many lower-risk procedures being performed during this timeframe. We show that surgery was, for the most part, safely conducted despite the risk of SARS-CoV-2 infection and postoperative complications. Various factors must be taken into account when allocating theatre resources for elective and non-urgent surgery during the pandemic.
The availability of nursing and surgical staff, limited ward space and decreased oxygen supply, as well as health-seeking behaviour of patients, have influenced surgical processes during the COVID-19 peaks. Yet, owing to the low risk of postoperative complications in our cohort, a fear of higher mortality in well-selected elective surgery was not justified. This specific concern should therefore receive lower priority when considering theatre resource allocation in future waves of the pandemic.

As previously mentioned, our lower 30-day mortality rate was similar to several international studies, including the experience of a UK trauma and orthopaedics unit where 206 trauma and urgent orthopaedic surgical procedures were performed during the peak of the pandemic (1 March - 31 May 2020). [10] Of these patients, $4.3 \%(n=9)$ died postoperatively; $55.5 \%$ of the deceased patients were SARS-CoV-2-negative, $1.45 \% \quad(n=3)$ were SARS-CoV-2-positive postoperatively, and 1 patient presented as asymptomatic and was not tested before surgery. The authors promote the usefulness of comparing and evaluating single-centre practice. A study that collated Northern Ireland regional data showed very low (1.9\%) overall 30 -day postoperative mortality in trauma and orthopaedic surgery patients. Of the patients, 5.5\% $(n=27)$ contracted SARS$\mathrm{CoV}-2$ perioperatively, and in this group the 30 -day mortality rate was $14.8 \%(n=4) \cdot{ }^{[11]}$ At the Nuffield Orthopaedic Centre in the UK, $9.4 \%$ of patients who underwent orthopaedic procedures as SARS-CoV-2-negative $(n=9)$ contracted SARS-CoV-2 within 30 days postoperatively. ${ }^{[12]}$ Of these patients, $66 \%$ ( $n=6)$ developed significant COVID-19related complications postoperatively, and there was a $33 \%(n=3) 30$-day mortality rate.
The overall 30-day mortality rate across the whole cohort of patients $(n=96)$ during this period was $3 \%(n=3)$. Another UK-based study reported an overall survival rate of $96 \%$ for patients undergoing emergency orthopaedic trauma surgery during the COVID-19 peak. $^{[13]}$ An Italian experience recorded an overall mortality of $4.6 \%(n=3)$ among fracture trauma patients. ${ }^{[14]}$ Of those who died, $5.8 \%(n=1)$ were SARS-CoV-2positive and $4.1 \%(n=2)$ were SARS-CoV2 -negative ( $p>0.05)$; 17 patients were SARSCoV-2-positive on admission or became positive within 14 days of admission. It is noteworthy that our perioperative SARSCoV-2 infection rate for patients we were able to obtain the data for was drastically lower than rates in these studies (Table 2).

Other international studies reported higher 30-day mortality and SARS-CoV-2 infection rates than those reported by this study. These included the COVIDSurg international cohort study, which reported a 30-day postoperative mortality rate of $28.8 \%(n=86 / 302)$ in patients undergoing orthopaedic procedures between 1 January and 31 March 2020. ${ }^{[5]}$ All these patients had SARS-CoV-2 infection confirmed within 7 days before or 30 days after surgery. In this orthopaedic patient group, $44.3 \%$ ( $n=131)$ exhibited postoperative pulmonary complications, defined as pneumonia, acute respiratory distress syndrome or unexpected postoperative ventilation. A UK-based retrospective cohort study reported an increased postoperative mortality rate of $30.5 \% \quad(n=25 / 82)$ in COVID-19-positive patients undergoing hip fracture surgery, compared with a rate of $10.3 \%(n=35 / 340)$ in COVID-19-negative patients $(p<0.001) .{ }^{[8]}$ In another fracture study conducted in New York, $12.3 \%$ of the patient cohort $(n=138)$ were COVID-19-positive and 10.1\% suspected COVID-19-positive. ${ }^{[9]}$ Increased mortality during hospitalisation and after 30 days (53\% of COVID-19-positive and $14 \%$ of suspected COVID-19 patients) was reported when compared with COVID-19negative patients (Table 2).

\section{Study limitations}

This study has limitations. During the study period, the COVID-19 swabbing criteria continued to evolve, and the clinical diagnosis of suspected COVID-19 patients was therefore not standardised. As previously mentioned, only COVID-19 PUI were swabbed for SARS-CoV-2 infection testing by quantitative PCR. We may have missed many negative COVID-19-screened, asymptomatic patients who were in fact positive for SARS-CoV-2 infection, yet this 


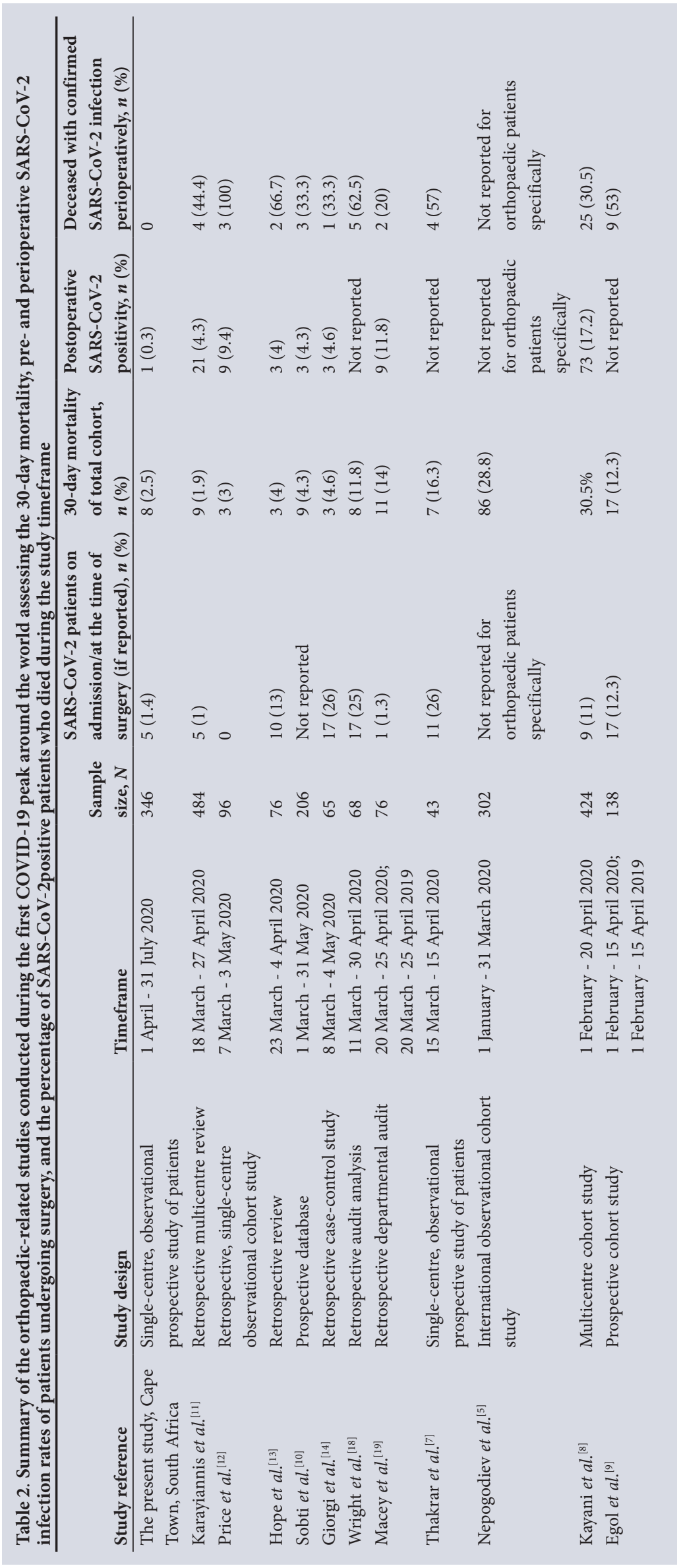

would not have changed our primary outcome of overall mortality. It is important to note that asymptomatic carriers can transmit the virus during the incubation phase, posing a risk to patients in the ward, and to nursing and surgical staff. ${ }^{[4]}$ Patient follow-up remains complex in the population serviced by our academic hospital. We were not able to successfully contact every patient who underwent surgery to assess their and their household contacts' COVID-19 status postoperatively, which would have given our data more strength and improved accuracy of reporting. In addition, several patients were LTFU because they did not attend their follow-up appointments. In our case, LTFU does not mean 'no death'. Although reporting of LTFU is quite rare in all the COVID survival-related studies to date, a study that excluded 21 patients and calculated the mortality percentage for the remaining patients reported a mortality rate of $5.4 \%,{ }^{[15]}$ while ours, for patients we were able to obtain 30-day mortality data for, was $2.5 \%$. If we were to assume that all 35 patients for whom we are missing data died, our mortality rate would equate to $10 \%$. This is still lower than the pooled prevalence mortality rate of $20 \%$ that took into account reports from UK, China, Turkey, India, Italy, Spain, the USA and France, ${ }^{[16]}$ and an overall mortality rate of $15 \%$ reported by a Canadian study. ${ }^{[17]}$ Our mortality rate, inclusive or exclusive of the missing data, falls within the globally reported rates. Further prospective studies with larger cohorts, comparing 30-day mortality data with previous years, and throughout the different COVID-19 peaks and in the presence of different SARS-CoV-2 variants, are needed for a deeper understanding of the risk associated with pre- and perioperative SARS-CoV-2 infection. In addition, we did not assess any postoperative complications in our SARS-CoV-2-positive patients.

\section{Conclusions}

We report a low 30-day mortality rate of $2.5 \%$ for patients undergoing orthopaedic surgery at our hospital during the first peak of the COVID19 pandemic in 2020 and a 30-day mortality rate of $0 \%$ for preoperatively confirmed SARSCoV-2-positive patients undergoing orthopaedic procedures. Of the 8 patients who died, 7 (87.5\%) underwent surgery for orthopaedic trauma. We can conclude that it was safe to operate on orthopaedic patients at our academic hospital during the first COVID-19 peak in SA. COVID-19 status and 30-day mortality data are important, as they will guide our surgeons on the most appropriate way to rapidly and safely resume elective and non-urgent surgery, with careful consideration of both patient and surgical staff risk.

Declaration. The research for this study was done in partial fulfulment of the requirements for RW's $\mathrm{PhD}$ degree at the University of Cape Town. 
Acknowledgements. We thank our research unit nurses Nomsa Yekiso and Nosipho Mncwabe for contacting patients telephonically, and Dr Walid Mugla for assisting with this. We also thank Moegammed Nasser Bardien for his efforts with pulling patient folders and Warren Hendricks for obtaining relevant patient data.

Author contributions. MH conceptualised the manuscript. RW extracted and prepared the data, accessed all databases, reviewed folders, prepared figures and wrote the manuscript. MH, ML, RDe, RDu, TH, WM, SR, $\mathrm{GMcC}, \mathrm{MN}$ and SM performed critical revision of the manuscript. All authors read and approved the final manuscript.

Funding. None.

Conflicts of interest. None.

1. South African Government News Agency. SA records close to 92\% COVID-19 recovery rate 6 November 2020. https://www.sanews.gov.za/south-africa/sa-records-close-92-covid-19-recoveryrate (accessed 11 December 2020).

2. Waters R, Dey R, Laubscher M, et al. Drastic reduction of orthopaedic services at an urban tertiary Waters R, Dey R, Laubscher M, et al. Drastic reduction of orthopaedic services at an urban tertiary
hospital in South Africa during COVID-19: Lessons for the future response to the pandemic. S Afr Med J 2021;111(3):240-244. https://doi.org/10.7196/SAMJ.2021.v111i3.15263

3. Prachand VN, Milner R, Angelos P, et al. Medically necessary, time-sensitive procedures: Scoring system to ethically and efficiently manage resource scarcity and provider risk during the COVID-19 pandemic. J Am Coll Surg 2020;231(2):281-288. https://doi.org/10.1016/j.jamcollsurg.2020.04.011

4. Lei S, Jiang F, Su W, et al. Clinical characteristics and outcomes of patients undergoing surgeries during the incubation period of COVID-19 infection. E Clin Med 2020;21:100331. https://doi.org/10.1016/j. eclinm.2020.100331

5. Nepogodiev D, Bhangu A, Glasbey JC, et al. Mortality and pulmonary complications in patients undergoing surgery with perioperative SARS-CoV-2 infection: An international cohort study. Lancet 2020;396(10243):27-38. https://doi.org/10.1016/S0140-6736(20)31182-X

6. Myles PS, Maswime S. Mitigating the risks of surgery during the COVID-19 pandemic. Lancet 2020;396(10243):2-3. https://doi.org/10.1016/S0140-6736(20)31256-3

7. Thakrar A, Chui K, Kapoor A, Hambidge J. Thirty-day mortality rate of patients with hip fractures Thakrar A, Chui K, Kapoor A, Hambidge J. Thirty-day mortality rate of patients with hip fractures
during the COVID-19 pandemic: A single centre prospective study in the United Kingdom. J Orthop during the COVID-19 pandemic: A single centre prospective study in the United
Trauma 2020;34(9):e325-e329. https://doi.org/10.1097/BOT.0000000000001889
8. Kayani B, Onochie E, Patil V, et al. The effects of COVID-19 on perioperative morbidity and mortality in patients with hip fractures: A multicentre cohort study. Bone Joint J 2020;102-B(9):1136-1145. in patients with hip fractures: A multicentre cohort study.

9. Egol KA, Konda SR, Bird ML, et al. Increased mortality and major complications in hip fracture care 9. Egol KA, Konda SR, Bird ML, et al. Increased mortality and major complications in hip fracture care
during the COVID-19 pandemic: A New York City perspective. J Orth Trauma 2020;34(8):395-402. during the COVID-19 pandemic: A New York

10. Sobti A, Memon K, Bhaskar RP, Unnithan A, Khaleel A. Analysis of mortality following trauma and orthopaedic surgery at the peak of COVID-19 pandemic. Br J Surg 2020;107(11):e472-e473. https:// doi.org/10.1002/bjs.11929

11. Karayiannis PN, Roberts V, Cassidy R, et al. 30-day mortality following trauma and orthopaedic surgery during the peak of the COVID-19 pandemic: A multicentre regional analysis of 484 patients. Bone Joint Open 2020;1(7):392-397. https://doi.org/10.1302/2633-1462.17.bjo-2020-0075.r1

12. Price A, Shearman AD, Hamilton TW, Alvand A, Kendrick B; COVID-19 NOC Surgical Team. 30-day outcome after orthopaedic surgery in patients assessed as negative for COVID-19 at the time of surgery during the peak of the pandemic. Bone Joint Open 2020;1(8):474-480. https://doi.org/10.1302/26331462.18.bjo-2020-0119.r1

13. Hope N, Gulli V, Hay D, et al. Outcomes of orthopaedic trauma patients undergoing surgery during the peak period of COVID-19 infection at a UK major trauma centre. Surgeon 2020 (epub 25 December 2020). https://doi.org/10.1016/j.surge.2020.11.009

14. Giorgi PD, Gallazzi E, Capitani P, et al. Mortality and morbidity in COVID-19 orthopedic trauma patients: Is early surgery the keystone? Pan Afr Med J 2021;38:163. https:// doi.org/10.11604/ pami.2021.38.163.27125

15. Clement ND, Hall AJ, Makaram NS, et al. IMPACT-Restart: The influence of COVID-19 on postoperative mortality and risk factors associated with SARS-CoV-2 infection after orthopaedic and trauma surgery. Bone Joint J 2020;102(12):1774-1781. https://doi.org/10.1302/0301-620x.102b12.bjj2020-1395.r2

16. Abate SM, Mantefardo B, Basu B. Postoperative mortality among surgical patients with COVID-19: A systematic review and meta-analysis. Patient Saf Surg 2020;14(1):1-4. https://doi.org/10.1186/ s13037-020-00262-6

17. Carrier FM, Amzallag É, Lecluyse V, et al. Postoperative outcomes in surgical COVID-19 patients: A multicenter cohort study. BMC Anesthesiol 2021;21(1):1-8. https://doi.org/10.1186/s12871-02101233-9

18. Wright EV, Musbahi O, Singh A, Somashekar N, Huber CP, Wiik AV. Increased perioperative mortality for femoral neck fractures in patients with coronavirus disease 2019 (COVID-19): Experience from the United Kingdom during the first wave of the pandemic. Patient Saf Surg 2021;15(1):1-8. https:// doi.org/10.1186/s13037-020-00279-x

19. Macey AR, Butler J, Martin SC, Tan TY, Leach WJ, Jamal B. 30-day outcomes in hip fracture patients during the COVID-19 pandemic compared to the preceding year. Bone Joint Open 2020;1(7):415-419. https://doi.org/10.1302/2633-1462.17.bjo-2020-0077.r

Accepted 1 June 2021. 